\title{
No conformal anomaly in unimodular gravity
}

\author{
Enrique Álvarez ${ }^{1,2, *}$ and Mario Herrero-Valea ${ }^{2, \dagger}$ \\ ${ }^{1}$ Physics Department, Theory Unit, CERN 1211 Genève 23, Switzerland \\ ${ }^{2}$ Instituto de Física Teórica UAM/CSIC and Departamento de Física Teórica, \\ Universidad Autónoma de Madrid, E-28049 Madrid, Spain
}

(Received 23 January 2013; published 23 April 2013)

\begin{abstract}
The conformal invariance of unimodular gravity survives quantum corrections, even in the presence of conformal matter. Unimodular gravity can actually be understood as a certain truncation of the full Einstein-Hilbert theory, where in the Einstein frame the metric tensor has unit determinant. Our result is compatible with the idea that the corresponding restriction in the functional integral is consistent as well.
\end{abstract}

PACS numbers: $04.50 . \mathrm{Kd}$

\section{INTRODUCTION}

A radical approach towards explaining why (the zero mode of) the vacuum energy seems to violate the equivalence principle (the active cosmological constant problem) is just to eliminate the direct coupling in the action between the potential energy and the gravitational field [1]. This leads us to consider unimodular theories, where the metric tensor is constrained to be unimodular

$$
g_{E} \equiv\left|\operatorname{det} g_{\mu \nu}^{E}\right|=1
$$

in the Einstein frame. This equality only stands in those reference frames obtained from the Einstein one by an area preserving diffeomorphism. Those are by definition the ones that enjoy unit Jacobian, and $g$ is a singlet under them.

We shall represent the absolute value of the determinant of the metric tensor in an arbitrary frame as $g$ instead of $|g|$ in order to simplify the corresponding formulas. We work in arbitrary dimension $n$ in order to be able to employ dimensional regularization as needed. The simplest nontrivial such unimodular action [1] reads

$$
\begin{aligned}
S_{U} \equiv & -M^{n-2} \int d^{n} x R_{E}+S_{\text {matt }} \\
= & -M^{n-2} \int d^{n} x g^{\frac{1}{n}}\left(R+\frac{(n-1)(n-2)}{4 n^{2}} \frac{g^{\mu \nu} \nabla_{\mu} g \nabla_{\nu} g}{g^{2}}\right) \\
& +S_{\text {matt }},
\end{aligned}
$$

where the $n$-dimensional Planck mass is related to the $n$-dimensional Newton constant through

$$
M^{n-2} \equiv \frac{1}{16 \pi G}
$$

and $S_{\text {matt }}$ is the matter contribution to the action.

This theory is conformally (Weyl) invariant under

$$
\tilde{g}_{\mu \nu}=\Omega^{2}(x) g_{\mu \nu}(x)
$$

\footnotetext{
*enrique.alvarez@uam.es

†mario.herrero@estudiante.uam.es
}

(the Einstein metric is inert under those) as well as under area preserving (transverse) diffeomorphisms, id est, those that enjoy unit Jacobian, thereby preserving the Lebesgue measure. We shall speak always of conformal invariance in the above sense.

The aim of this paper is to explore whether this gauge symmetry is anomalous or survives when one-loop quantum corrections are taken into account. The result we have found is that, given the fact that this theory can be thought of as a partial gauge fixed sector of a conformal upgrading of general relativity, there is no conformal anomaly for unimodular gravity, even when conformal matter is included.

Other interesting viewpoints on the cosmological constant from the point of view of unimodular gravity are presented in Refs. [2,3]. In this last reference Smolin suggested the absence of conformal anomaly for related theories.

We will proceed as follows. First, we will define a more general scalar-tensor theory by introducing a spurion field $\sigma$. This theory is diffeomorphism as well as conformal invariant and unimodular gravity is no more than a partial gauge fixed sector of it. This happens to be, also, the same theory that 't Hooft proposed [4] in order to solve some special issues of black hole complementarity. Consequently, in Sec. III we will explore 't Hooft's approach in order to obtain the divergent part of the oneloop effective action of such theory. In Sec. IV, however, we will show how the scalar-tensor action can be written in a more useful and manifestly conformal invariant form and we will use it to easily compute the one-loop gravitational counterterm in Sec. V. Our result is not only consistent with 't Hooft's computations but also shows in a very clear way how the anomaly vanishes.

\section{A MORE GENERAL SCALAR-TENSOR THEORY}

It is technically quite complicated to gauge fix a theory invariant under area preserving (transverse) diffeomorphisms only, because the theory is reducible, id est, the corresponding gauge parameters are not independent. 
ENRIQUE ÁLVAREZ AND MARIO HERRERO-VALEA

This usually demands a huge ghost sector [5]. In order to avoid these, presumably physically irrelevant intricacies, it proves convenient to introduce a new theory which enjoys diffeomorphism invariance and that is such that the unimodular theory is a partial gauge fixing of it. This is easily achieved by introducing a compensating field, $C(x)$, defined so that

$$
g(x) C^{2} \equiv e^{\frac{2 n}{\sqrt{(n-1)(n-2)}} \sigma(x)}
$$

transforms as a true scalar. This diffeomorphism invariant theory is still Weyl invariant provided that

$$
e^{\frac{2 n}{\sqrt{(n-1)(n-2)}} \tilde{\sigma}(x)}=\Omega^{2 n} e^{\frac{2 n}{\sqrt{(n-1)(n-2)}} \sigma(x)} .
$$

Id est, the composite exponential field has conformal weight $-2 n$. In general, a conformal tensor of conformal weight $-\lambda$ behaves under conformal transformations as

$$
\delta T=\lambda T .
$$

At the linear level, $\Omega(x) \equiv 1+\omega(x)$, the spurion field $\sigma$ transforms with the gauge parameter like a true Goldstone boson does:

$$
\delta \sigma=\sqrt{(n-1)(n-2)} \omega .
$$

This result conveys the fact that the spurion is nothing else than the dilaton. The unimodular theory of our interest is recovered when the partial unitary gauge

$$
C=1
$$

is chosen. The residual gauge symmetries are then the area preserving diffeomorphisms as well as Weyl invariance.

Let us be quite explicit on this point. Under a diffeomorphism

$$
\delta x^{\mu}=\xi^{\mu}
$$

the compensating field behaves as

$$
\delta C=\partial_{\lambda} \xi^{\lambda} C-\xi^{\lambda} \partial_{\lambda} C,
$$

whereas it is a singlet under conformal transformations. Under finite transformations

$$
C^{\prime}\left(x^{\prime}\right)=C(x) \cdot \operatorname{det} \frac{\partial x^{\prime}}{\partial x} .
$$

To reach the gauge $C=1$ starting from a nonvanishing $C \neq 0$ it is then enough to choose

$$
\operatorname{det}\left(\frac{\partial x^{\prime}}{\partial x}\right)=\frac{1}{C(x)} .
$$

The gauge $C=1$ means that

$$
e^{\frac{2 n}{\sqrt{(n-1)(n-2)}} \sigma(x)}=g(x) .
$$

PHYSICAL REVIEW D 87, 084054 (2013)

The new action is then written as

$$
\begin{aligned}
S \equiv & \int d^{n} x \sqrt{g}\left\{e ^ { - \sqrt { \frac { n - 2 } { n - 1 } } \sigma } \left[-M^{n-2}\left(R+g^{\mu \nu} \nabla_{\mu} \sigma \nabla_{\nu} \sigma\right)\right.\right. \\
& \left.\left.+\frac{1}{2} g^{\mu \nu} \nabla_{\mu} \Phi \nabla_{\nu} \Phi\right]-e^{-\frac{n}{\sqrt{(n-1)(n-2)}} \sigma} V(\Phi)\right\} .
\end{aligned}
$$

This action is conformal invariant as well as diffeomorphism invariant with all fields transforming as indicated above.

The spurion $\sigma(x)$ corresponds to a conformal rescaling of the metric, and behaves as a ghost (its kinetic energy term has the wrong sign). It is standard, since the work [6], to perform its functional integral over imaginary values of the field. We shall keep an open mind on this issue for the time being.

The canonically normalized field is

$$
\phi_{g} \equiv-2 \sqrt{2} M^{\frac{n-2}{2}} \sqrt{\frac{n-1}{n-2}} e^{-\frac{1}{2} \sqrt{\frac{n-2}{n-1}} \sigma} .
$$

The old gauge $C=1$ now reads

$$
\phi_{g}+2^{\frac{3}{2}} M^{\frac{n-2}{2}} \sqrt{\frac{n-1}{n-2}} g^{-\frac{n-2}{4 n}}=0 .
$$

In terms of $\phi_{g}$ the action is

$$
\begin{aligned}
S_{\mathrm{ST}}= & \int d^{n} x \sqrt{g}\left\{-\frac{n-2}{8(n-1)} R \phi_{g}^{2}-\frac{1}{2} g^{\mu \nu} \nabla_{\mu} \phi_{g} \nabla_{\nu} \phi_{g}\right. \\
& +\frac{n-2}{8(n-1) M^{n-2}} \phi_{g}^{2} \frac{1}{2}(\nabla \Phi)^{2} \\
& \left.-(-1)^{\frac{2 n}{n-2}}\left(\frac{n-2}{8(n-1)}\right)^{\frac{n}{n-2}} \frac{1}{M^{n}} \phi_{g}^{\frac{2 n}{n-2}} V(\Phi)\right\} .
\end{aligned}
$$

It is instructive to study in detail how the equations of motion (EM) of the scalar-tensor theory reduce to the unimodular ones in the unitary gauge. Indeed,

$\frac{\delta S_{U}}{\delta g_{\mu \nu}}=\frac{\delta S_{\mathrm{ST}}}{\delta g_{\mu \nu}}+\left.\frac{\delta S_{\mathrm{ST}}}{\delta \phi_{g}} \frac{\delta \phi_{g}}{\delta g_{\mu \nu}}\right|_{\phi_{g}=-2^{\frac{3}{2}} M^{\frac{n-2}{2}} \sqrt{\frac{n-1}{n-2}} g^{-n-2-2}}$.

This conveys the fact that the scalar-tensor EM imply the unimodular EM, whereas the converse assertion is untrue: the unimodular EM do not imply the scalar-tensor ones. The unimodular theory is a subsector of the more general scalar tensor theory, as stated in the title of the present section.

In this scalar-tensor theory it is possible to go to the Einstein frame through

$$
g_{\mu \nu}=2^{\frac{6}{n-2}} M^{2}\left(\frac{n-1}{n-2}\right)^{\frac{2}{n-2}} \phi_{g}^{-\frac{4}{n-2}} g_{\mu \nu}^{E} .
$$

This metric $g_{\mu \nu}^{E}$ is a conformal singlet; that is, it remains invariant under Weyl transformations. In the gauge $C=1$ we go round the whole circle and the metric in Einstein's frame is unimodular, 


$$
\{C=1\} \Rightarrow g_{E}=1 \text {. }
$$

It is also possible here to define, again following [4,6],

$$
i \log \phi_{g} \equiv \eta \text {. }
$$

In the following we will just work with the gravitational sector, since as we will show later, inclusion of matter will not change any of our conclusions. Thus, in the rest of this text we will forget about the scalar field.

\section{III. 'T HOOFT'S APPROACH: EFFECTIVE ACTION AFTER INTEGRATING THE CONFORMAL FACTOR}

The gravitational piece of the previous Lagrangian is identical to the one proposed by 't Hooft in Ref. [4] in order to solve conceptual problems of black holes. For this purpose it is essential to integrate first over the scalar field in such a way as to get a conformally invariant theory of gravity. The divergent part of the functional integral over the scalar field can be easily computed after it is conveniently rotated to imaginary values, as advertised earlier

$$
e^{-\frac{1}{2} \sqrt{\frac{n-2}{n-1}} \sigma(x)} \equiv 1+i \alpha(x) \quad \alpha \in \mathbb{R}
$$

and the result of this integration over $\mathcal{D} \alpha$ can be expressed in terms of the Weyl tensor. Let us revisit its origin. The Schouten tensor is defined as

$$
A_{\alpha \beta} \equiv \frac{1}{n-2}\left(R_{\alpha \beta}-\frac{1}{2(n-1)} R g_{\alpha \beta}\right)
$$

and the Weyl tensor reads

$$
\begin{aligned}
W_{\alpha \beta \mu \nu} \equiv & R_{\alpha \beta \mu \nu}+\left(A_{\beta \mu} g_{\alpha \nu}+A_{\alpha \nu} g_{\beta \mu}\right. \\
& \left.-A_{\beta \nu} g_{\alpha \mu}-A_{\alpha \mu} g_{\beta \nu}\right) .
\end{aligned}
$$

Under conformal transformations, it transforms as a conformal tensor of weight $\lambda=-1$ :

$$
\tilde{W}_{\alpha \beta \mu \nu} \equiv e^{2 \sigma} W_{\alpha \beta \mu \nu} .
$$

So its square has weight $\lambda=2$ in such a way that

$$
\begin{aligned}
& |g|^{2 / n} W^{\mu \nu \rho \sigma} W_{\mu \nu \rho \sigma} \\
& \quad=|g|^{2 / n}\left(R_{\mu \nu \rho \sigma} R^{\mu \nu \rho \sigma}-2 R_{\mu \nu} R^{\mu \nu}+\frac{1}{3} R^{2}\right)
\end{aligned}
$$

is pointwise invariant (but behaves as a true scalar in four dimensions only).

The Weyl tensor vanishes identically in low dimension $n=2$ and $n=3$ and a space with $n \geq 4$ is conformally flat if and only if $W=0$. In that case, the claim is that the counterterm reads

$$
L_{\mathrm{div}}=-\frac{\sqrt{g}}{480 \pi^{2}(n-4)} W_{\mu \nu \rho \sigma} W^{\mu \nu \rho \sigma} .
$$

This functional behavior (barring the coefficient) could have been predicted from the fact that the resulting theory had to be pointwise conformal invariant. It can also be written as

$$
L_{\mathrm{div}}=\frac{\sqrt{g}}{960 \pi^{2}(n-4)}\left(R_{\mu \nu} R^{\mu \nu}-\frac{1}{3} R^{2}\right) .
$$

The second expression is easily obtained assuming that the Euler topological invariant vanishes, id est

$$
\int d^{4} x \sqrt{g}\left(R_{\mu \nu \rho \sigma} R^{\mu \nu \rho \sigma}-4 R_{\mu \nu} R^{\mu \nu}-R^{2}\right)=0 .
$$

It is perhaps worth remarking that the quantity

$$
\delta\left(g^{\frac{2}{n}} W_{\mu \nu \rho \sigma} W^{\mu \nu \rho \sigma}\right)=0
$$

which is invariant under area preserving diffeomorphisms only, enjoys pointwise conformal invariance in any dimension; when the power of the determinant is determined in order to enhance area preserving diffeomorphisms to the full group of diffeomorphisms, the resulting expression is conformal invariant only in dimension $n=4$

$\delta\left(\sqrt{g} W_{\mu \nu \rho \sigma} W^{\mu \nu \rho \sigma}\right)=-\frac{4-n}{2 n} 2 n \omega(x) \sqrt{g} W_{\mu \nu \rho \sigma} W^{\mu \nu \rho \sigma}$.

This fact, first noticed by Duff [7] leads to the understanding of the standard conformal anomaly in dimensional regularization through finite remainders coming from the $\epsilon \frac{1}{\epsilon}$ cancellation.

\section{CONFORMAL INVARIANCE}

Instead of working with the scalar-tensor theory in the form we just obtained, let us clarify its physical content by defining the following vector field:

$W_{\mu} \equiv \frac{1}{n-2} e^{-\sqrt{\frac{n-2}{n-1}} \sigma} \nabla_{\mu} e^{\sqrt{\frac{n-2}{n-1}} \sigma}=\frac{1}{\sqrt{(n-2)(n-1))}} \nabla_{\mu} \sigma$

which under conformal transformations behaves as an Abelian gauge field

$$
W_{\mu}^{\prime}=\Omega^{-1} \nabla_{\mu} \Omega+W_{\mu} .
$$

This fact encodes a deep meaning, namely that in general we should be always able to construct a pointwise invariant conformal theory from a noninvariant one by adding interactions with this gauge field in a similar way as it is done in a Yang-Mills theory to implement local invariance under $S U(N)$ to the fermionic matter. This is precisely the situation we have in the unimodular theory (which is more clear when described through this more general scalartensor theory), which naively, and forgetting for the moment the implications of the $C=1$ partial gauge fixing, is no more than an upgrading of Einstein-Hilbert theory into a conformal invariant one, so it has to be possible to rewrite it just as general relativity coupled to this $W_{\mu}$ field. 
Thus, let us start as usual by defining a gauge covariant derivative by meanings of the gauge connection, which upgrades the Riemannian connection to

$$
\Gamma(W)_{\nu \rho}^{\mu}=\Gamma_{\nu \rho}^{\mu}-\delta_{\nu}^{\mu} W_{\rho}-\delta_{\rho}^{\mu} W_{\nu}+g_{\nu \rho} W^{\mu}
$$

which allows us to define a conformal (as well as diffeomorphism) covariant derivative by

$$
D_{\mu} T=\nabla_{\mu}^{\Gamma(W)} T+\lambda W_{\mu} T,
$$

where $-\lambda$ is the conformal weight of the tensor $T$ and $\nabla_{\mu}^{\Gamma(W)}$ states for the derivative defined through the Weyl connection $\Gamma(W)$.

The important fact that arises here is that even if this Weyl connection is not a metric one, all dynamical quantities can however be canonically constructed just by defining a new metric in such a way that

$G_{\alpha \beta}=e^{-\frac{2 \sigma}{\sqrt{(n-2)(n-1)}}} g_{\alpha \beta} \rightarrow \Gamma(W)_{\nu \rho}^{\mu}\left[g_{\alpha \beta}\right]=\Gamma_{\nu \rho}^{\mu}\left[G_{\alpha \beta}\right]$,

which enjoys all expected properties.

So at this point things are straightforward and we can compute naive Weyl invariant (once proper integration measure is provided) geometrical quantities out of the $D_{\mu}$ derivative, such as the Riemann tensor defined by its conmutator, which will be related to the ones computed just with the usual metric $g_{\mu \nu}$ in a fancy way. We consign details of those computations to the Appendix but just let us recall the final result for the Weyl curvature scalar in terms of the usual one together with the spurion field, which is

$$
\mathcal{R}=R-2 \sqrt{\frac{n-1}{n-2}} \nabla^{2} \sigma-(\nabla \sigma)^{2}
$$

The success of this construct is that, via an integration by parts, it corresponds exactly with the Lagrangian density of the scalar-tensor theory, so the full action can be rewritten in a manifestly Weyl invariant way as

$S=\int d^{n} x \sqrt{G} \mathcal{R}=\int d^{n} x \sqrt{g} e^{-\sqrt{\frac{n-2}{n-1}} \sigma}\left(R+(\nabla \sigma)^{2}\right)$

and this shows clearly how the Weyl invariant scalar-tensor theory is just a completion of the usual Einstein-Hilbert theory in order to have pointwise conformal invariance through the gauge field $W_{\mu}$.

It is also interesting to check what the partial gauge fixing $C=1$ means with respect to conformal invariance. From Eq. (2.1), we can see that it reduces to just $G=1$, which is exactly the unimodularity condition that we also imposed in the Einstein-Hilbert Lagrangian to define the unimodular theory. This clearly shows that this theory, at least at the classical level, is no more than a common partially gauge fixed sector of both general relativity and conformal gravity, corresponding to those physical systems that, maintaining conformal invariance (which implies the impossibility of adding a cosmological constant term to the Lagrangian), have general coordinate transformation invariance reduced to area preserving diffeomorphisms only.

This statement has also another useful implication, which is that when written through the conformally invariant metric $G_{\mu \nu}$, the background field expansion of the action is straightforward and identical to the expansion of the Einstein-Hilbert Lagrangian with the added step of changing all geometrical quantities by the ones constructed through $W_{\mu}$. This is easily understood since the covariant structure is the same in both cases and the only difference is the adding of conformal invariance.

\section{ONE-LOOP COMPUTATION}

Our goal in this work was to determine whether the conformal invariance of unimodular gravity was broken by quantum corrections in the form of a trace anomaly.

There is a general issue of consistency here.

When computing anomalies, the problem is usually reduced to a theory propagating in a background (nondynamical) gravitational field. This gives rise to the computation of determinants that depend upon the background metric. What we are doing in this paper is slightly different, in the sense that we are considering the gravitational field as a dynamical entity, and computing its one-loop effects. It is a fact that the Einstein-Hilbert Lagrangian is nonrenormalizable. This has been shown to be the case also for the unimodular variants, as studied in Ref. [1]. The consistency of our approach is then not guaranteed. The meaning of our result is then rather that no obvious inconsistency appears when considering the theory to one-loop order. This fact alone is highly nontrivial.

As it is explained in Appendix B, the computation of the conformal anomaly can be reduced to the calculation of the $n=d$ Schwinger-de Witt coefficient in the expansion of the heat kernel corresponding to the quadratic differential operator of the effective action for quantum fluctuations. However, when the expression (4.7) is taken into account, things are easier, since what the heat kernel expansion states is that the conformal (or trace) anomaly is

$$
-\int d(\mathrm{vol}) T=\lambda a_{d}
$$

where $-\lambda$ is the conformal weight of the corresponding second order operator, $T \equiv T_{\mu \nu} g^{\mu \nu}$ is the trace of the oneloop energy-momentum tensor, and $a_{d}$ is a certain coefficient in the expansion of the heat kernel of the operator of quadratic fluctuations as given in the Appendix, formula (B8). So if we are dealing with pointwise conformal operators in our Lagrangian, this vanishes identically and computing the Schwinger-de Witt coefficient is not necessary. And, recalling what we proved before, this is exactly the situation we are dealing with, so we should expect the conformal anomaly to cancel in this theory. However, 
allow us to be more explicit and compute the counterterm exactly by recalling that the full action of the unimodular theory in the scalar-tensor description was written in a manifestly conformally (Weyl) invariant way, namely

$$
\begin{aligned}
S & =-M^{n-2} \int d^{n} x \sqrt{G} \mathcal{R} \\
& =-M^{n-2} \int d^{n} x \sqrt{g} e^{-\sqrt{\frac{n-2}{n-1}} \sigma}\left(R+(\nabla \sigma)^{2}\right) .
\end{aligned}
$$

Therefore, performing a background field expansion (which has been discussed in some detail in the second reference of Ref. [1])

$$
g_{\mu \nu} \equiv \bar{g}_{\mu \nu}+h_{\mu \nu} \quad \sigma \equiv \bar{\sigma}+\sigma
$$

provided with the (often dubbed classical) conformal transformations

$$
\begin{aligned}
\delta_{C} \bar{g}_{\mu \nu} & =2 \omega(x) \bar{g}_{\mu \nu} \quad \delta_{C} h_{\mu \nu}=2 \omega(x) h_{\mu \nu} \\
\delta_{C} \bar{\sigma} & =\sqrt{(n-1)(n-2)} \omega \quad \delta_{C} \sigma=0
\end{aligned}
$$

we can reconstruct again the conformal invariant structure, this time at the linear level, by expanding all quantities in the same way as we did in Sec. IV, but using this time the background field, so we will denote everything computed this way by adding a bar over it. The fact that the variation of the dynamical spurion $\sigma$ vanishes means that all the expressions of Weyl invariant geometrical quantities will be identical to the ones at the nonlinear level by just replacing the full field $\sigma$ by the background ${ }^{1}$ one $\bar{\sigma}$ and since all these changes can be encoded, as we showed before, into a conformal rescaling of the metric, this implies that the perturbative expansion of this action will match the well-known one of the Einstein-Hilbert Lagrangian with just the corresponding change of metric and operators done at every step. So doing it and taking care of fixing the gauge in a conformally (Weyl) background invariant way, ${ }^{2}$ we are done.

The background (zeroth order) term reads simply

$$
\bar{S}=-M^{n-2} \int d^{n} x \sqrt{\bar{g}} e^{-\sqrt{\frac{n-2}{n-1}} \bar{\sigma}} \overline{\mathcal{R}} .
$$

On the other hand, the linear terms that have to cancel in order to ensure absence of tadpoles are

$$
S_{\sigma}=-M^{n-2} \int d^{n} x \sqrt{\bar{g}} e^{-\sqrt{\frac{n-2}{n-1}} \bar{\sigma}} \sqrt{\frac{n-2}{n-1}} \overline{\mathcal{R}} \sigma,
$$

\footnotetext{
${ }^{1}$ It is worth remarking that doing this, the covariant derivative of the gravitational fluctuation $h_{\mu \nu}$, which is a tensor of conformal weight $\lambda=-2$, transforms as another conformal tensor of the same weight.

${ }^{2}$ The best option, taking into account that our goal is to compute the gravitational one-loop counterterm, is generalizing the harmonic gauge to $\bar{D}_{\mu} h^{\mu \nu}=0$.
}

$$
S_{h}=M^{n-2} \int d^{n} x \sqrt{\bar{g}} e^{-\sqrt{\frac{n-2}{n-1}} \bar{\sigma}} \sqrt{\frac{n-2}{n-1}} \bar{\epsilon}_{\mu \nu} h^{\mu \nu},
$$

where $\bar{\epsilon}_{\mu \nu}$ is the background Einstein tensor and we have performed a convenient partial integration in the gravitational fluctuation action. The linear equations of motion for the background metric are then encoded into these linear terms and read

$$
-\bar{R}_{\alpha \beta}+\frac{1}{2} \bar{R} \bar{g}_{\alpha \beta}=\bar{\nabla}_{\alpha} \bar{\sigma} \bar{\nabla}_{\beta} \bar{\sigma}-\frac{1}{2}(\bar{\nabla} \bar{\sigma})^{2} \bar{g}_{\alpha \beta} .
$$

The trace of the above implies directly $\bar{R}=-(\bar{\nabla} \bar{\sigma})^{2}$ and on the other hand, the geometrical Bianchi identities demand that

$$
\begin{aligned}
0 & =\bar{\nabla}^{\alpha}\left(-\bar{R}_{\alpha \beta}+\frac{1}{2} \bar{R} \bar{g}_{\alpha \beta}\right) \\
& =\bar{\nabla}^{2} \bar{\sigma} \bar{\nabla}_{\beta} \bar{\sigma}=\frac{1}{2} \sqrt{\frac{n-2}{n-1}} \bar{\nabla}_{\beta} \bar{\sigma}\left((\bar{\nabla} \bar{\sigma})^{2}-\bar{R}\right) .
\end{aligned}
$$

Altogether they imply $\bar{R}=(\bar{\nabla} \bar{\sigma})^{2}=\bar{\nabla}^{2} \bar{\sigma}=0=\overline{\mathcal{R}}$, which, as with Einstein equations, is no more than a consequence of the background equations of motion once we take into account the substitution of operators by conformal ones that we were discussing.

Finally and as we argued, the second order term has to be the same as in the expansion of the Einstein-Hilbert Lagrangian, where at each step the substitution

$$
\bar{g}_{\mu \nu} \rightarrow \bar{G}_{\alpha \beta}=e^{-\frac{2}{\sqrt{(n-2)(n-1)}} \bar{\sigma}} \bar{g}_{\alpha \beta}
$$

is made, which implies also substituting all derivatives by the background Weyl invariant one $\bar{D}_{\mu}$.

Thus

$$
\begin{aligned}
S_{h^{2}}= & -M^{n-2} \int d^{n} x \sqrt{\bar{G}}\left[\frac{1}{4} \bar{D}^{\mu} H \bar{D}_{\mu} H-\frac{1}{2} \bar{D}_{\mu} H \bar{D}^{\rho} H_{\rho}^{\mu}\right. \\
& +\frac{1}{2} \bar{D}_{\mu} H^{\mu \rho} \bar{D}_{\nu} H_{\rho}^{\nu}-\frac{1}{4} \bar{D}_{\mu} H^{\nu \rho} \bar{D}^{\mu} H_{\nu \rho}-\overline{\mathcal{R}}_{\nu \beta} H_{\alpha}^{\beta} H^{\nu \alpha} \\
& \left.+\frac{1}{2} H \overline{\mathcal{R}}_{\alpha \beta} H^{\alpha \beta}-\frac{\overline{\mathcal{R}}}{2}\left(\frac{H^{2}}{4}-\frac{1}{2} H^{\alpha \beta} H_{\alpha \beta}\right)\right]
\end{aligned}
$$

where $H_{\mu \nu}$ is the graviton fluctuation of the rescaled metric $G_{\mu \nu}$, corresponding to

$$
H_{\mu \nu}=e^{-\frac{2}{\sqrt{(n-2)(n-1)}} \bar{\sigma}}\left(h_{\mu \nu}-\frac{2 \sigma \bar{g}_{\mu \nu}}{\sqrt{(n-2)(n-1)}}\right) .
$$

This in turn means that (provided that the corresponding conformal harmonic gauge fixing is used) the counterterm is simply given in terms of the 't Hooft-Veltman [4] counterterm by performing the same operator substitution we were doing formerly 


$$
\begin{aligned}
S_{c}= & \frac{1}{8 \pi^{2}(n-4)} \frac{203}{80} \int d^{n} x \sqrt{\bar{G}} \overline{\mathcal{R}}^{2} \\
= & \frac{1}{8 \pi^{2}(n-4)} \frac{203}{80} \int d^{n} x \sqrt{\bar{g}} e^{-\sqrt{\frac{n-2}{n-1}} \bar{\sigma}} \\
& \times\left(\bar{R}-2 \sqrt{\frac{n-1}{n-2}} \bar{\nabla}^{2} \bar{\sigma}-(\bar{\nabla} \bar{\sigma})^{2}\right)^{2},
\end{aligned}
$$

which is manifestly pointwise conformally invariant and also it vanishes on shell when background equations of motion are taken into account. This is in accord with the naive fact that the conformal anomaly should vanish owing to the manifest conformal invariance of the action.

The inclusion of noninteracting conformal matter does not change the situation. For example, a scalar field interacts with the gravitational field according to

$$
\begin{aligned}
S_{\text {matt }} & \equiv \int d^{n} x \frac{1}{2} g_{E}^{\mu \nu} \nabla_{\mu} \Phi \nabla_{\nu} \Phi \\
& =\int d^{n} x g^{\frac{1}{n}} \frac{1}{2} g^{\mu \nu} \nabla_{\mu} \Phi \nabla_{\nu} \Phi .
\end{aligned}
$$

Once embedded in a diffeomorphism invariant theory, the action principle reads

$$
S=\int d^{n} x \sqrt{\bar{g}} e^{-\sqrt{\frac{n-2}{n-1}} \bar{\sigma}} \frac{1}{2} \bar{g}^{\mu \nu} \bar{\nabla}_{\mu} \Phi \bar{\nabla}_{\nu} \Phi
$$

and given the transformation of $\bar{\sigma}$, it is plain to check that the operator

$$
\Delta f \equiv \bar{\nabla}_{\mu}\left(\sqrt{\bar{g}} e^{-\sqrt{\frac{n-2}{n-1}} \bar{\sigma}} \bar{g}^{\mu \nu} \bar{\nabla}_{\mu} f\right)
$$

is conformally invariant.

\section{CONCLUSIONS}

It has been shown that the conformal invariance of unimodular gravity survives quantum corrections, even in the presence of scalar conformal matter. This result is a consequence of the fact that the corresponding operator governing quadratic fluctuations around an arbitrary background is manifestly conformal invariant (vanishing conformal weight).

Another way of looking at this result is through the computation of the counterterm, which is quite simply determined from the standard 't Hooft-Veltman counterterm. This counterterm is Weyl invariant for any dimension, id est, its variation vanishes as opposed to being proportional to $n-4$. It actually vanishes on shell, once the background equations of motion are used. The fact that the conformal anomaly should vanish for unimodular gravity was already conjectured by Blas in his Ph.D. thesis work [8].

The physical situation is not unlike the gauge current in a vectorlike gauge theory, where it is also quite plain that no anomaly is present.

As a general remark, the unimodular theory can be understood as a certain truncation of the full EinsteinHilbert theory, where in a certain frame (the Einstein frame) the metric tensor is unimodular (with determinant equal to one). Our result is compatible with the idea that the corresponding restriction at the quantum level (i.e., in the functional integral) is consistent as well.

\section{ACKNOWLEDGMENTS}

We have enjoyed many discussions with Luis AlvarezGaumé. This work has been partially supported by the European Union FP7 ITN INVISIBLES (Marie Curie Actions, PITN- GA-2011- 289442) and (HPRN-CT-20000148) as well as by FPA2009-09017 (DGI del MCyT, Spain) and S2009ESP-1473 (CA Madrid). M. H. acknowledges a "Campus de Excelencia" grant from the Departamento de Física Teórica of the UAM. The authors acknowledge the support of the Spanish MINECO's "Centro de Excelencia Severo Ochoa" Programme under Grant No. SEV-2012-0249.

\section{APPENDIX A: WEYL COVARIANT CURVATURE}

Once the Weyl covariant derivative defined through the gauge field $W_{\mu}$ is constructed, geometrical quantities can be computed. To start with, the commutator of two of such derivatives defines a curvature through Ricci's identity (and is independent of the conformal weight of the tensor acted upon, so the apparently arbitrary term $\lambda W_{\mu} T$ does not cause any contradiction and indeed it is needed to ensure that the derivative of the metric vanishes)

$$
\begin{aligned}
\mathcal{R}_{\mu \nu \rho \sigma}= & R_{\mu \nu \rho \sigma}-g_{\mu \rho}\left(\nabla_{\nu} W_{\sigma}+W_{\nu} W_{\sigma}\right)-g_{\mu \sigma}\left(\nabla_{\nu} W_{\sigma}+W_{\nu} W_{\rho}\right)+g_{\nu \rho}\left(\nabla_{\mu} W_{\sigma}+W_{\mu} W_{\sigma}\right)+g_{\nu \sigma}\left(\nabla_{\mu} W_{\rho}+W_{\mu} W_{\rho}\right) \\
& +\left(\nabla_{\lambda} W^{\lambda}\right)^{2}\left(g_{\mu \sigma} g_{\nu \rho}-g_{\mu \rho} g_{\nu \sigma}\right) \\
= & R_{\mu \nu \rho \sigma}+g_{\mu \rho}\left(\nabla_{\nu} \nabla_{\sigma} \sigma+\nabla_{\nu} \sigma \nabla_{\sigma} \sigma\right)-g_{\mu \sigma}\left(\nabla_{\nu} \nabla_{\sigma} \sigma+\nabla_{\nu} \sigma \nabla_{\rho} \sigma\right) \\
& -g_{\nu \rho}\left(\nabla_{\mu} \nabla_{\sigma} \sigma+\nabla_{\mu} \sigma \nabla_{\sigma} \sigma\right)+g_{\nu \sigma}\left(\nabla_{\mu} \nabla_{\rho} \sigma+\nabla_{\mu} \sigma \nabla_{\rho} \sigma\right)+(\nabla \sigma)^{2}\left(g_{\mu \sigma} g_{\nu \rho}-g_{\mu \rho} g_{\nu \sigma}\right) .
\end{aligned}
$$

It is easy to realize that, defining a new metric by a conformal rescaling $G_{\alpha \beta}=e^{-\frac{2 \sigma}{\sqrt{(n-2)(n-1)}}} g_{\alpha \beta}$, what we have is

$$
\mathcal{R}_{\mu \nu \rho \sigma}=e^{\frac{2}{\sqrt{(n-1)(n-2)}} \sigma} R_{\mu \nu \rho \sigma}\left[g_{\alpha \beta} e^{-\frac{2}{\sqrt{(n-1)(n-2)}} \sigma}\right]=\left(\frac{G}{g}\right)^{1 / n} R_{\mu \nu \rho \sigma}\left[G_{\alpha \beta}\right]
$$


which corresponds to the usual Riemann tensor that we would compute using the metric $G_{\alpha \beta}$ with a prefactor $(G / g)^{1 / n}$ whose origin is to ensure pointwise conformal invariance. Accordingly

$$
\begin{aligned}
\mathcal{R}_{\mu \nu}= & R_{\mu \nu}+(n-2)\left(\nabla_{\mu} W_{\nu}+W_{\mu} W_{\nu}\right) \\
& +g_{\mu \nu}\left(\nabla_{\lambda} W^{\lambda}-(n-2) W_{\lambda} W^{\lambda}\right) \\
= & R_{\mu \nu}-\sqrt{\frac{n-2}{n-1} \nabla_{\mu} \nabla_{\nu} \sigma+\frac{1}{n-1} \nabla_{\mu} \sigma \nabla_{\nu} \sigma} \\
& -g_{\mu \nu}\left(\frac{1}{\sqrt{(n-1)(n-2)}} \nabla^{2} \sigma+\frac{1}{n-1} \nabla_{\lambda} \sigma \nabla^{\lambda} \sigma\right) .
\end{aligned}
$$

And this Ricci tensor also has a quite simple interpretation

$$
\begin{aligned}
& \mathcal{R}_{\mu \nu}=e^{\frac{2}{\sqrt{(n-1)(n-2)}} \sigma} R_{\mu \nu}\left[g_{\alpha \beta} e^{-\frac{2}{\sqrt{(n-1)(n-2)}} \sigma}\right] \\
& =\left(\frac{G}{g}\right)^{1 / n} R_{\mu \nu}\left[G_{\alpha \beta}\right]
\end{aligned}
$$

manifestly conformal invariant under

$g_{\mu \nu} \rightarrow \Omega^{2} g_{\mu \nu} \quad e^{-\frac{2}{\sqrt{(n-1)(n-2)}} \sigma} \rightarrow \Omega^{-2} e^{-\frac{2}{\sqrt{(n-1)(n-2)}} \sigma}$.

From this, the curvature scalar is straightforward and inherits the same interpretation

$$
\begin{aligned}
\mathcal{R} & =R+2(n-1) \nabla_{\lambda} W^{\lambda}-(n-2)(n-1) W_{\lambda} W^{\lambda} \\
& =R-2 \sqrt{\frac{n-1}{n-2}} \nabla^{2} \sigma-(\nabla \sigma)^{2}=\left(\frac{G}{g}\right)^{1 / n} R\left[G_{\alpha \beta}\right] .
\end{aligned}
$$

From all this, the Einstein tensor results to be

$$
\begin{aligned}
\mathcal{E}_{\mu \nu}= & R_{\mu \nu}-\frac{1}{2} R g_{\mu \nu}+\frac{1}{n-1} \nabla_{\mu} \sigma \nabla_{\nu} \sigma \\
& +\sqrt{(n-1)(n-2)} \nabla^{2} \sigma g_{\mu \nu}+\frac{n-3}{2(n-1)}(\nabla \sigma)^{2} g_{\mu \nu} .
\end{aligned}
$$

Finally, taking into account that the measure

$$
\sqrt{g} e^{-\frac{n}{\sqrt{(n-1)(n-2)}} \sigma} d^{n} x=\sqrt{G}\left(\frac{G}{g}\right)^{-1 / n}
$$

is conformal invariant, the only dimension-two pointwise invariant operator is

$$
\int d^{n} x \sqrt{g} e^{-\frac{n}{\sqrt{(n-1)(n-2)}} \sigma} R=\int d^{n} x \sqrt{g} e^{-\sqrt{\frac{n-2}{n-1}} \sigma}\left(R+(\nabla \sigma)^{2}\right)
$$

and after integration by parts, the full action can then be written as

$$
S=\int d^{n} x \sqrt{g} e^{-\frac{n}{\sqrt{(n-1)(n-2)}} \sigma} \mathcal{R}=\int d^{n} x \sqrt{G} \mathcal{R},
$$

where the factors $G / g$ cancel exactly and show how dynamics can be obtained from the metric $G_{\alpha \beta}$ even if it does not encode all information about the nature of the Weyl covariant derivative (explicitly, it knows nothing about the $\lambda W_{\mu} T$ term of the derivative).

At the linear level, the conformal classical (or background) transformations are

$$
\begin{aligned}
\delta_{C} \bar{g}_{\mu \nu} & =2 \omega(x) \bar{g}_{\mu \nu} \quad \delta_{C} h_{\mu \nu}=2 \omega(x) h_{\mu \nu} \\
\delta_{C} \bar{\sigma} & =\sqrt{(n-1)(n-2)} \omega \quad \delta_{C} \sigma=0
\end{aligned}
$$

and since they vanish for the spurion field fluctuation, this means that all the geometrical construct we just carried out in this appendix can be redone on the background field expansion as well just by replacing $\sigma$ by $\bar{\sigma}$.

\section{APPENDIX B: CONFORMAL ANOMALY}

It is well known that one-loop computations are equivalent to the calculation of functional determinants. One of the simplest definitions of the determinant of an operator is through the zeta-function technique [9]. We shall follow conventions as in Ref. [10]. Given a differential operator of the general form

$$
\Delta \equiv-D_{\mu} D^{\mu}+Y
$$

with $D_{\mu} \equiv \partial_{\mu}+X_{\mu}$, we assume that the elliptic operator $\Delta$ enjoys eigenvalues $\lambda_{n}$

$$
\Delta \phi_{n}=\lambda_{n} \phi_{n}
$$

normalized in such a way that

$$
\int d^{n} x \sqrt{g} \phi_{i} \phi_{j}=\delta_{i j} .
$$

Now the heat kernel is formally defined as

$$
K(\tau) \equiv e^{-\tau \Delta}
$$

and its action on functions reads

$$
(K f)(x)=\int d(\mathrm{vol})_{y} K(x, y ; \tau) f(y) .
$$

The ultraviolet (UV) behavior is controlled by the short time Schwinger-de Witt expansion which reads

$$
K(x, y ; \tau)=K_{0}(x, y ; \tau) \sum_{p=0} b_{2 p} \tau^{p},
$$

where for instance the flat space kernel reads

$$
K_{0}(x, y ; \tau)=\frac{1}{(4 \pi \tau)^{\frac{n}{2}}} e^{-\frac{(x-y)^{2}}{4 \tau}}
$$

The integrated quantity $Y(\tau, f) \equiv \operatorname{tr}(K f)$ also enjoys a corresponding short time expansion

$$
Y(\tau, f)=\sum_{k=0} \tau^{\frac{k-n}{2}} a_{k}(f) .
$$


ENRIQUE ÁLVAREZ AND MARIO HERRERO-VALEA

The trace in the preceding formulas involves spacetime integration as well as sum over all finite rank indices. Sometimes one simply writes $Y(\tau) \equiv Y(\tau, 1)$.

The zeta function is defined as

$$
\Gamma(s) \zeta(s)=\int_{0}^{\infty} d t t^{s-1} Y(t)=\sum_{n} \lambda_{n}^{-s},
$$

where the second equality is even more formal than the first one.

The determinant of the differential operator is then defined [9] as

$$
\operatorname{det} \Delta \equiv \prod_{n} \lambda_{n} \equiv e^{-\zeta^{\prime}(0)} .
$$

Now assume that we have a quantum field theory that we dimensionally regularize, id est, we make $n=d+\epsilon$, where $d$ is the physical dimension (for example $d=4$ ), then, at the one-loop level, there is a divergent piece in the effective action

$$
W_{\infty}=-\left.\frac{1}{2} \log \operatorname{det} \Delta\right|_{\infty}=-\mu^{\epsilon} \frac{a_{d}}{\epsilon} .
$$

On the other hand, when performing a rigid Weyl transformation on the spacetime metric

$$
\tilde{g}_{\mu \nu}=\Omega^{2} g_{\mu \nu}=(1+2 \omega) g_{\mu \nu}
$$

the eigenvalues of the operator transform in a definite manner which coincide with the conformal weight $\lambda$ of the operator:

$$
\widetilde{\lambda_{n}} \equiv \Omega^{-\lambda} \lambda_{n}
$$

Usually the conformal weight is just the mass dimension of the operator in the sense of dimensional analysis.

According to Erdmenger [11] a conformal covariant operator $D$ transforms under local (not only rigid) Weyl transformations in such a way that there exist two numbers $(a, b)$ such that the Weyl rescaled operator is given by

$$
\tilde{D} \phi=\Omega^{-b} D\left(\Omega^{a} \phi\right) .
$$

It follows that the new eigenfunctions are given by

$$
\tilde{\phi}_{n}=\Omega^{-a} \phi_{n}
$$

and the new eigenvalues by

$$
\tilde{\lambda}_{n}=\Omega^{-b} \lambda_{n}
$$

The archetype of such operators is the conformal Laplacian

$$
\Delta_{c} \equiv \Delta-\frac{1}{4} \frac{n-2}{n-1} R
$$

which is such that

$$
\bar{\Delta}_{c}\left(\Omega^{-\frac{n-2}{2}} \phi\right)=\Omega^{-\frac{n+2}{2}} \Delta \phi .
$$

There are no known diffeomorphism invariant operators built out of the metric alone with $b=0$.
PHYSICAL REVIEW D 87, 084054 (2013)

In the case of the standard scalar Laplacian,

$$
\Delta \equiv \nabla^{2} \equiv \frac{1}{\sqrt{g}} \partial_{\mu}\left(g^{\mu \nu} \sqrt{g} \partial_{\nu}\right)
$$

the conformal weight coincides with its mass dimension, $\lambda=2$.

The new zeta function after the Weyl transformation is given in general by

$$
\tilde{\zeta}(s)=\Omega^{D s} \zeta(s)
$$

so that the determinant defined through the zeta function scales as

$$
\operatorname{det} \tilde{\Delta}=\Omega^{-\lambda \zeta(0)} \operatorname{det} \Delta
$$

and this modifies correspondingly the effective action

$$
\tilde{W}=W+\lambda \omega \zeta(0) .
$$

The energy-momentum tensor is defined in such a way that under a general variation of the metric the variation of the effective action reads

$$
\delta W \equiv \frac{1}{2} \int d(\operatorname{vol})_{x} T_{\mu \nu} \delta g^{\mu \nu}
$$

which in the particular case that this variation is proportional to the metric tensor itself (like in a conformal transformation at the linear level), $\delta g^{\mu \nu}=-2 \omega g^{\mu \nu}$ yields the integrated trace of the energy-momentum tensor

$$
\delta W=-\int d(\operatorname{vol}) \omega T .
$$

Conformal invariance in the above sense then means that the energy-momentum tensor must be traceless. When quantum corrections are taken into account, it follows that

$$
-\int d(\mathrm{vol}) T=\lambda \zeta(0)
$$

It is not difficult to show that

$$
\zeta(0) \equiv \lim _{s \rightarrow 0} s \int_{0}^{\infty} d t t^{s-1} Y(t)=\lim _{s \rightarrow 0} s \int_{0}^{1} d t t^{s-1} Y(t)=a_{d},
$$

where $n=d$ is the specific value of the spacetime dimension. The conformal anomaly is usually then written as

$$
-\int d(\mathrm{vol}) T=\lambda a_{d} \text {. }
$$

The Schwinger-de Witt coefficient corresponding to the physical dimension, $n=d$, precisely coincides with the divergent part of the effective action when computed in dimensional regularization as indicated above. This means that in order to compute the one-loop conformal anomaly in many cases it is enough to compute the corresponding counterterm. 
This argument shows clearly that when the conformal weight of the operator of interest vanishes, $\lambda=0$, all eigenvalues remain invariant and there is no conformal anomaly for determinants defined through the zeta function. In our case this will follow from the manifest Weyl invariance of the construction of the operator at all steps. This conformal invariance in turn is inherited from the mother theory which enjoys invariance under area preserving diffeomorphisms only. This is the origin of the background dilaton $\bar{\sigma}$ of gravitational origin, which is essential in our approach.
[1] E. Alvarez and A.F. Faedo, Phys. Rev. D 76, 064013 (2007); E. Alvarez and M. Herrero-Valea, J. Cosmol. Astropart. Phys. 01 (2013) 014.

[2] Y. J. Ng and H. van Dam, J. Math. Phys. (N.Y.) 32, 1337 (1991).

[3] L. Smolin, Phys. Rev. D 80, 084003 (2009).

[4] G. 't Hooft, arXiv:1009.0669.

[5] M. Henneaux and C. Teitelboim, Quantization of Gauge Systems (Princeton University, Princeton, NJ, 1992), p. 520.

[6] G. W. Gibbons, S. W. Hawking, and M. J. Perry, Nucl. Phys. B138, 141 (1978).
[7] M.J. Duff, Classical Quantum Gravity 11, 1387 (1994).

[8] D. Blas, arXiv:0809.3744.

[9] S. W. Hawking, Commun. Math. Phys. 55, 133 (1977).

[10] E. Alvarez and A.F. Faedo, J. High Energy Phys. 05 (2006) 046; E. Alvarez, A. F. Faedo, and J. J. LopezVillarejo, J. High Energy Phys. 10 (2008) 023.

[11] J. Erdmenger, Classical Quantum Gravity 14, 2061 (1997).

[12] L.P. Eisenhart, Non-Riemannian Geometry (Dover, New York, 2005). 